\title{
THE FIRST EXIT OF ALMOST STRONGLY RECURRENT SEMI-MARKOV PROCESSES
}

Abstract. Let $\stackrel{n}{X}(\cdot), n \in \mathbb{N}$, be a sequence of homogeneous semi-Markov processes (HSMP) on a countable set $K$, all with the same initial p.d. concentrated on a non-empty proper subset $J$. The subrenewal kernels which are restrictions of the corresponding renewal kernels $\stackrel{n}{\mathbf{Q}}$ on $K \times K$ to $J \times J$ are assumed to be suitably convergent to a renewal kernel $\mathbf{P}$ (on $J \times J$ ). The HSMP on $J$ corresponding to $\mathbf{P}$ is assumed to be strongly recurrent. Let $\left[\pi_{j} ; j \in J\right]$ be the stationary p.d. of the embedded Markov chain. In terms of the averaged p.d.f. $F_{\vartheta}(t):=\sum_{j, k \in J} \pi_{j} P_{j, k}(t), t \in \mathbb{R}_{+}$, and its Laplace-Stieltjes transform $\widetilde{F}_{\vartheta}$, the above assumptions imply:

The time $\stackrel{n}{T}_{J}$ of the first exit of $\stackrel{n}{X}(\cdot)$ from $J$ has a limit p.d. (up to some constant factors) iff $1-\widetilde{F}_{\vartheta}$ is regularly varying at 0 with a positive degree, say $\alpha \in(0,1]$. Then the transform of the limit p.d.f. equals $\widetilde{G}^{(\alpha)}(s)=$ $\left(1+s^{\alpha}\right)^{-1}, \operatorname{Re} s \geq 0$.

This extends the results by V. S. Korolyuk and A. F. Turbin (1976) obtained for $\alpha=1$ under essentially stronger conditions.

1. Introduction. The time $T$ till the first "failure" of a modern exploitation system, during regular service, can be modeled by the random sum of random variables

$$
T=\vartheta_{1}+\ldots+\vartheta_{\nu}
$$

where w.p. 1 (with probability 1 ),

$\nu \in \mathbb{N}:=\{0,1, \ldots\} \quad$ and $\quad \vartheta_{m} \in \mathbb{R}_{+}:=[0, \infty)$ for $m \in \mathbb{N}_{1}:=\{1,2, \ldots\}$.

1991 Mathematics Subject Classification: Primary 60K15, 60K20; Secondary 60F05.

Key words and phrases: semi-Markov, Markov renewal, recurrent Markov processes; first exit; limit distribution; extended exponential p.d. 
The non-negative r.v. (random variable) $\vartheta_{m}$ represents the length of the $m$ th time interval elapsing between consecutive passages in the state space of the system.

In most cases, a satisfactory approximation is obtained already under the assumption that

$$
\vartheta=\left(\vartheta_{m} ; m \in \mathbb{N}_{1}\right) \text { is i.i.d. }
$$

(i.e. $\vartheta$ consists of independent identically distributed variables), or equivalently that

$$
\tau=\left(\tau_{m}:=\sum_{l=1}^{m} \vartheta_{l} ; m \in \mathbb{N}\right) \text { is a simple renewal process. }
$$

Usually, an additional assumption is made: that the "failures" can appear all with the same probability at each instant of $\tau$, independently of each other and independently of $\tau$. More precisely, that

$$
\begin{gathered}
\tau \text { and } \nu \text { are independent, } \\
\operatorname{Pr}\{\nu=m\}=(1-\varepsilon)^{m} \varepsilon \quad \text { for } m \in \mathbb{N} .
\end{gathered}
$$

In cases important for applications, the value of $\varepsilon$ is a rather small positive number. Therefore, for practical calculations, the limit p.d.f. (probability distribution function) of $T$ (suitably normalized by a constant factor as $\varepsilon \rightarrow 0^{+}$) can be used instead of its exact p.d.f. $F_{T}$.

Obviously, the smaller $\varepsilon$ the greater the number of terms in (1). Correspondingly, some violations in (1)-(4) become admissible. For instance, as for regenerative processes, the first and last few terms in (1) can obey a different p.d. than the other distances between elements of $\tau$, without affecting the limit p.d. A wide list of such violations due to the nature of real processes can be found e.g. in [10]. One can look at these results as extensions of a result by J. Keilson [4], which asserts the limit p.d. of $\varepsilon T$ to be exponential whenever the (common) expectation of $\vartheta_{m}$ is positive and finite. For a wide review of results related to reliability theory and/or queueing theory, the reader is referred e.g. to I. B. Gertsbakh [3] and S. Asmussen [1].

In a general setting one can admit the (common) p.d.f. $F_{\vartheta}$ of $\vartheta_{m}$ to vary with $\varepsilon$. Necessary and sufficient conditions for the existence and characterization of a proper limit p.d.f. of (the normalized) $T$ are given by A. D. Solovyev [9].

In our problems, the p.d.f. $F_{\vartheta}$ remains "almost constant" as $\varepsilon \rightarrow 0^{+}$, and the independence of the p.d.f. $F_{\vartheta}$ of $\varepsilon$ becomes a pattern case. This special assumption allows us to reformulate the result of [9] in terms of the regular variability of $F_{\vartheta}$. For the sake of completeness, these facts are presented as Theorem 1 and Proposition 1 in Section 2. We stress that even under (1)-(4) with $F_{\vartheta}$ independent of $\varepsilon$ we have: 
- the finiteness of the expectation of the distances collected in $\vartheta$ is not necessary for the existence of the exponential limit p.d. of $T$;

- in some cases the limit p.d. of $T$ is not exponential.

More precisely, the Laplace-Stieltjes transform of the limit p.d.f. has to equal

$$
\widetilde{G}^{(\alpha)}(s):=\left(1+s^{\alpha}\right)^{-1} \quad \text { for } s \geq 0, \text { where } \alpha \in(0,1] .
$$

Moreover, for every $0<\alpha \leq 1$ there is $F_{\vartheta}$ such that the limit equals $\widetilde{G}^{(\alpha)}$. The limits can be seen as an extension of the class of exponential distributions, admitting the exponent $\alpha$ to be less than 1 .

Our main goal is to prove similar statements for a class of semi-Markov (or Markov renewal) processes with at most countable state space. Then the r.v. $T$ is the time elapsing till the first exit from a subset $J$ of the state space and $F_{\vartheta}$ is a suitable stationary average of the p.d.f. of the distances between the instants of renewals within $J$ (cf. Sections 6-7).

Under our assumptions formulated in Section 7, the semi-Markov processes approach a regenerative one. Therefore, the main result (Theorem 2 in Section 7) can probably be derived directly from Theorem 1, or through some coupling procedure. However, the evaluation of the norming scale factor and/or the interpretation of $\varepsilon$ might become unreadable or tedious. Therefore we are following the main steps of V. S. Korolyuk and A. F. Turbin [6], who have given a very natural use of perturbed stochastic matrices for the solution of the renewal equation related to this problem. The formulas obtained here cover some new examples, which fall outside the scope of [6], even under the finiteness of the expectations of the distances between renewals.

In order to give a complete outline of semi-Markov processes, we have developed a suitable matrix calculus in Sections 3-4, and presented a short review of the basic notions in Sections 5-6 (see also [8]).

Let us close this section with a list of some notation. For any measurable function $f: \mathbb{R}_{+} \rightarrow X$ with values in a Banach space $X$, integrable as follows:

$$
\|f \exp (-a \cdot)\|_{L\left(\mathbb{R}_{+}, X, m\right)}<\infty, \quad \text { where } a \in \mathbb{R},
$$

the Laplace transform of $f$ with respect to $m$ is defined by

$$
\mathcal{L}_{m}(f)(s):=\int_{\mathbb{R}_{+}} f(t) \exp (-s t) d m(t) \quad \text { for } \operatorname{Re} s \geq a \text { (at least). }
$$

The notation is used independently of whether $m$ means a measure or a d.f. of locally bounded variation on $\mathbb{R}_{+}$. A suitable extension of the operations to matrices is introduced in Section 3.

If $m$ denotes the Lebesgue measure, then the subscript is omitted.

For $f=1$ we add simply a tilde over the symbol denoting the measure (or its d.f.). The result is called the Laplace-Stieltjes transform of $m$. In 
particular, for any p.d.f. $F$ on $\mathbb{R}_{+}$we have

$$
\widetilde{F}(s)= \begin{cases}\int_{\mathbb{R}_{+}} \exp (-s t) d F(t)=\mathcal{L}_{F}(1)(s) & \text { for } \operatorname{Re} s \geq 0, \\ s \int_{\mathbb{R}_{+}} \exp (-s t) F(t) d t=s \mathcal{L}(F)(s) & \text { for } \operatorname{Re} s>0 .\end{cases}
$$

We use the standard properties of the transformation and regularly varying functions as given e.g. in the books by W. Feller [2] and E. Seneta [7]. Accordingly, without essential losses, in most of our proofs, the independent variable of the transforms is restricted to real numbers. In particular, writing $s \rightarrow 0^{+}$we mean $s>0$ and $s \rightarrow 0$.

2. The case of simple renewal processes. Conditions (1)-(4) imply that the transform $\widetilde{F}_{T}$ of the p.d.f. $F_{T}$ of $T$ can be evaluated as follows:

$$
\begin{aligned}
\widetilde{F}_{T}(s) & =\sum_{n=0}^{\infty} \operatorname{Pr}\{\nu=m\}\left(\widetilde{F}_{\vartheta}(s)\right)^{m} \\
& =\left(1+\frac{1-\varepsilon}{\varepsilon}\left(1-\widetilde{F}_{\vartheta}(s)\right)\right)^{-1} \quad \text { for } s>0,
\end{aligned}
$$

where $F_{\vartheta}$ stands for the (common) p.d.f. of the distances between the renewals. Following the main steps of Theorem 2 of [2, Section XIII.6] and Lemma 2 of [2, Section VIII.8] we come to

THEOREM 1. Let $\stackrel{n}{T}$ be given by $(1)-(4)$, or by $(9)$, with $\varepsilon=\varepsilon_{n}$ satisfying (a)

$$
\varepsilon_{n} \rightarrow 0^{+} \text {and } \varepsilon_{n+1} / \varepsilon_{n} \rightarrow 1 \quad \text { as } n \rightarrow \infty,
$$

and suppose that the p.d.f. $F_{\vartheta}$ (the same for every $n$ ) satisfies

(b) $\quad \operatorname{supp} F_{\vartheta} \subset \mathbb{R}_{+}, \quad 1>F_{\vartheta}(0) \quad\left(=\operatorname{Pr}\left\{\vartheta_{m}=0\right\}\right.$ for $\left.m \in \mathbb{N}_{1}\right)$.

(i) The following conditions are equivalent:

- for some constants $\left(c_{n}>0 ; n \in \mathbb{N}\right)$ the p.d.f.'s of $c_{n} \stackrel{n}{T}$ tend to a p.d.f. not concentrated on $\{0\}$ as $n \rightarrow \infty$;

- $F_{\vartheta}$ is in the strict domain of attraction of a strictly stable p.d.f. on $\mathbb{R}_{+}$which is not concentrated on $\{0\}$;

- $1-\widetilde{F}_{\vartheta}$ is regularly varying at 0 with a positive degree.

(ii) Under any one of the conditions of (i), there are $\alpha \in(0,1]$ and $c>0$ such that

- $\widetilde{F}_{c_{n} T}(s) \rightarrow\left(1+c s^{\alpha}\right)^{-1}$ as $n \rightarrow \infty$ for $\operatorname{Re} s \geq 0$;

- $\left(\widetilde{F}_{\vartheta}\left(c_{n} s\right)\right)^{\varepsilon_{n}^{-1}} \rightarrow \exp \left(-c s^{\alpha}\right)$ as $n \rightarrow \infty$ for $\operatorname{Re} s \geq 0$;

- the exponent of regularity of $1-\widetilde{F}_{\vartheta}$ at 0 equals $\alpha$.

The conditions can be characterized by Tauberian theorems as follows. 
Proposition 1 [2, Chapters VIII and XIII]. For every p.d.f. $F$ on $\mathbb{R}_{+}$ and every $\alpha \in(0,1)$, the following conditions are equivalent:

(i) $s^{-\alpha}(1-\widetilde{F}(s))$ is slowly varying as $s \rightarrow 0^{+}$;

(ii) $t^{\alpha-1} \int_{0}^{t}(1-F(u)) d u$ is slowly varying as $t \rightarrow \infty$;

(iii) $t^{\alpha}(1-F(t))$ is slowly varying as $t \rightarrow \infty$.

For $\alpha=1$, (i) and (ii) remain equivalent, but (iii) becomes essentially stronger than (i) and (ii).

Proof. By (6)-(8), the function $\widetilde{U}(s):=s^{-1}(1-\widetilde{F}(s))$, Re $s>0$, is the transform of

$$
U(t):= \begin{cases}\int_{0}^{t}(1-F(u)) d u, & t \in \mathbb{R}_{+}, \\ 0, & t<0 .\end{cases}
$$

In terms of $\varrho=1-\alpha \geq 0$, the equivalence of (i) and (ii) for $\alpha \in(0,1]$ is shown in Theorem 1 of [2, Section XIII.5]. The equivalence of (i) and (iii) for $\alpha \in(0,1)$ is given by Theorem 4 of [2, Section XIII.5], because the density $1-F$ of $U$ is monotone in $(0, \infty)$. In case of $\alpha=1$, it suffices to note that $U(t)=\int_{0}^{t} u^{-p} Z(u) d u=: Z_{p}(t)$ with $p=1$, where $Z(t):=t(1-F(t))$ is slowly varying if (iii) is assumed. Then, referring to the Lemma of $[2$, Section VIII.9] we infer that $U$ is regularly varying with degree $p+1=0$, i.e. (ii) is satisfied.

To finish the proof, we consider $F(t):=\max \{0 ; 1-\exp (-[\log t])\}$, where $[x]$ stands for the integer part of $x$. Then $F$ does not satisfy (iii) since the ratio

$$
(1-F(t))^{-1}(1-F(\sqrt{e} \cdot t))=\exp ([\log t]-[\log t+0.5])
$$

is not convergent as $t \rightarrow \infty$ (since it is periodic with respect to $\log t$ ). On the other hand, for $t \geq e$, the function of (ii) with $\alpha=1$ equals $U$ and is piecewise linear with vertices at $\left(e^{n},(n-1)(e-1)\right) \in \mathbb{R}_{+}^{2}, n \in \mathbb{N}_{1}$. Thus, for $c \geq 1$ and $t>e^{2}$, we have

$$
1 \leq(U(t))^{-1} U(c t) \leq([\log t]-1)^{-1}[\log t+\log c],
$$

where the right hand side tends to 1 as $t \rightarrow \infty$.

Remark 1 . For every $\alpha \in(0,1]$, the transform of the strongly stable p.d.f. of exponent $\alpha$ equals $\exp \left(-s^{\alpha}\right)$ and satisfies conditions (i) of Theorem 1. Also, according to Proposition 1, the Pareto d.f. equal to $1-t^{\alpha}$, $t>1$, satisfies conditions (i) of Theorem 1. Therefore, if substituted for $F_{\vartheta}$, they cause the transform $\widetilde{F}_{c_{n} T}(s)$ to tend to $\left(1+s^{\alpha}\right)^{-1}$ for some $\left(c_{n} ; n \in \mathbb{N}\right)$. Note that the mean of the Pareto distributions is equal to $\infty$ for all $\alpha$ not exceeding 1. However, for $\alpha=1$ the limit p.d. of $c_{n} \stackrel{n}{T}$ is exponential. For a characterization of limits with arbitrary dependence of $F_{\vartheta}$ on $\varepsilon$ the reader is referred to [9], and for a review, to [3]. 
The degree of regularity can be expressed in terms of the moment's $d . f$. of $F$ given by

$$
F^{(l)}(t):=\int_{0}^{t} u^{l} d F(u), \quad t \in \mathbb{R}_{+} .
$$

Namely, introducing the $l$ th order moment of $F$ and $l$ th order upper fractional derivative of $\widetilde{F}$ at 0 by the equalities

$$
f^{(l)}:=F^{(l)}(\infty), \quad \delta^{(l)} \tilde{f}:=\limsup _{s \rightarrow 0^{+}} s^{-l}(1-\widetilde{F}(s)),
$$

for a p.d.f. regularly varying according to (i) of Proposition 1, we have

$$
\alpha=\sup \left\{l: 0 \leq l<1, f^{(l)}<\infty\right\}=\sup \left\{l: 0 \leq l<1, \delta^{(l)} \tilde{f}<\infty\right\} .
$$

3. Matrix operations. In what follows, for any pair of at most countable sets $J$ and $K$ and a Banach space $\langle X ;|| X\rangle$, we denote by $\left\langle X^{[J \times K]} ;\|\|_{X}\right\rangle$ the Banach space of all $X$-valued $(J, K)$-matrices $\mathbf{x}=$ $\left[x_{j, k} ;(j, k) \in J \times K\right]$ bounded with respect to the matrix norm

$$
\|\mathbf{x}\|_{X}:=\sup _{j \in J}\left\{\sum_{k \in K}\left|x_{j, k}\right|_{X}\right\} .
$$

For $L \subset J$ and $M \subset K$, the symbol $\mathbf{x}_{L, M}$ denotes the $(L, M)$-submatrix of $\mathbf{x}$. Thus, the above matrix satisfies the equality $\mathbf{x}=\mathbf{x}_{J, K}$.

Any function given by a list of values, as $\left(y_{j} ; j \in J\right) \in X^{J}$, is identified with a suitable $J$-row; here $\mathbf{y}=\left[y_{j} ;(i, j) \in \sharp \times J\right]$, where $\sharp$ stands for the dummy one-element set. Accordingly, the transposed matrix $\mathbf{z}=\mathbf{y}^{\prime} \in$ $X^{J \times \sharp}$ is a $J$-column. The restriction of the domain of rows and columns is indicated by a suitable position of the separating comma, as in the equalities $\mathbf{y}=\mathbf{y}_{, L}$ and $\mathbf{z}=\mathbf{z}_{L}$. Note that $X^{[J \times \sharp]}$ and the space of uniformly bounded sequences $l^{\infty}(J, X)$ are isometric. Also, $X^{[\sharp \times J]}$ and the space of summable sequences $l^{1}(J, X)$ are isometric. If $X$ is the field of real numbers, then the corresponding spaces are denoted simply by $l^{\infty}(J)$ and $l^{1}(J)$, respectively. We assume that $\circ: X \times Y \rightarrow Z$ is a bilinear operator bounded as follows:

$$
|x \circ y|_{Z} \leq|x|_{X} \cdot|y|_{Y}
$$

Then, for $\mathbf{x} \in X^{[J \times K]}$ and $\mathbf{y} \in Y^{[K \times L]}$, the matrix product can be defined as follows:

$$
\mathbf{x} \circ \mathbf{y}:=\left[\sum_{k \in K} x_{j, k} \circ y_{k, l} ;(j, l) \in J \times L\right] \in Z^{[J \times L]},
$$

and by inequalities (13), the product is also bounded:

$$
\|\mathbf{x} \circ \mathbf{y}\|_{Z} \leq\|\mathbf{x}\|_{X} \cdot\|\mathbf{y}\|_{Y} .
$$


Some statements of this work become more readable if expressed in terms of the partial matrix product (which is also an element of $Z^{[J \times L]}$ ), defined as follows:

$$
\mathbf{x} \stackrel{M}{\circ} \mathbf{y}:=\mathbf{x}_{J, M} \circ \mathbf{y}_{M, L} \quad \text { for } M \subset K .
$$

The 0 symbol denotes the zero of any space of elements of matrices, and 1 denotes any neutral factor, left or right, whenever it exists. Correspondingly, $\mathbf{0}_{J, K}, \mathbf{I}_{J, K}$ and $\mathbf{1}_{J, K}$ denote the zero-matrix, the matrix of 1 's, and the unit matrix restricted to $J \times K$, respectively. The last convention means that

$$
\mathbf{1}_{J, K}:=\left[\delta_{j, k} \cdot 1 ;(j, k) \in J \times K\right],
$$

where $\delta_{j, k}$ is the Kronecker symbol, and the dot stands for multiplication by scalars. Matrices with elements proportional to the neutral factor are equivalently treated as matrices of scalars.

If $\left\langle X,||_{X}, \circ, 1\right\rangle$ is an associative Banach algebra, then

$$
\left\langle X^{[J \times J]},\|\|_{X}, \circ, \mathbf{1}_{J, J}\right\rangle
$$

is also such an algebra. The powers of an element $\mathbf{x}$ of the latter algebra are written as

$$
\mathbf{x}^{\circ n}:=\underbrace{\mathbf{x} \circ \ldots \circ \mathbf{x}}_{n \text { factors }} \quad \text { for } n \in \mathbb{N}_{1} .
$$

We extend this assuming $\mathbf{x}^{\circ 0}:=\mathbf{1}_{J, J}$. For invertible $\mathbf{x}, \mathbf{x}^{\circ(-n)}:=\left(\mathbf{x}^{\circ(-1)}\right)^{n}$.

Definition 1. A sequence $(\stackrel{n}{\mathbf{x}} ; n \in \mathbb{N}) \subset X^{[J \times K]}$ is said to be matrixweakly convergent (mw-convergent) to $\mathbf{x} \in X^{[J \times K]}$ if for every $\mathbf{a} \in l^{1}(J)$ and $\mathbf{b} \in l^{\infty}(K)$, we have $|\mathbf{a} \cdot(\mathbf{x}-\stackrel{n}{\mathbf{x}}) \cdot \mathbf{b}|_{X} \rightarrow 0$ (in $\left.X\right)$ as $n \rightarrow \infty$.

Equivalently, for uniformly bounded sequences the condition requires that for every $\mathbf{b}$ as above, every element of the $J$-column $(\mathbf{x}-\stackrel{n}{\mathbf{x}}) \cdot \mathbf{b}$ tends to zero (in $X$ ) as $n \rightarrow \infty$.

The matrices of our concern consist of elements of the following commutative Banach algebras:

$$
\begin{aligned}
\mathcal{D}_{+}:=\left\{F: \mathbb{R}_{+} \rightarrow \mathbb{R}: \operatorname{supp} F \subset \mathbb{R}_{+}, F\right. \text { is right-continuous } \\
\text { and } \left.|F|_{\text {var }}<\infty\right\},
\end{aligned}
$$

where ||$_{\text {var }}$ stands for variation over $\mathbb{R}_{+}$, and

(20) $\mathcal{A}_{+}:=\left\{\varphi: \mathbb{C}_{+} \rightarrow \mathbb{C}: \varphi\right.$ is analytic in Int $\mathbb{C}_{+}$and continuous in $\mathbb{C}_{+}$,

$$
\text { and } \left.|\varphi|_{+}:=\sup \left\{|\varphi(s)|: s \in \mathbb{C}_{+}\right\}<\infty\right\} \text {. }
$$

The binary operations in these spaces are the convolution $*$ and the multiplication of functions $\bullet$, respectively. The neutral elements for them are the indicators of $\mathbb{R}_{+}$and $\mathbb{C}_{+}$, respectively. In particular, these binary operations are applicable for the matrix calculus we have sketched above. 
The (Laplace-Stieltjes) transformation $F \rightarrow \widetilde{F}$, defined by (8) with $s \in \mathbb{C}_{+}$, is an injective contracting homomorphism of the algebra $\mathcal{D}_{+}$into $\mathcal{A}_{+}$. The transform of a $\mathcal{D}_{+}$-valued matrix is defined as the $\mathcal{A}_{+}$-valued matrix consisting of the transforms of the corresponding entries of the original matrix. The matrix norm of the image does not exceed the matrix norm of the original and the Multiplication Rule for matrix convolution can be used. Thus, for $\mathbf{F} \in \mathcal{D}_{+}^{[J \times K]}$ and $\mathbf{G} \in \mathcal{D}_{+}^{[K \times L]}$, we have

$$
\|\widetilde{\mathbf{F}}\|_{+} \leq\|\mathbf{F}\|_{\text {var }}, \quad \mathbf{F}(\infty):=\lim _{t \rightarrow \infty} \mathbf{F}(t)=\widetilde{\mathbf{F}}(0), \quad(\mathbf{F} * \mathbf{G})^{\sim}=\widetilde{\mathbf{F}} \bullet \widetilde{\mathbf{G}}
$$

The transformation does not change the norm of any function from the following cone, which contains all p.d.f.'s on $\mathbb{R}_{+}$:

$$
\mathcal{D}_{+}^{\uparrow}:=\left\{F \in \mathcal{D}_{+}: F \text { is nondecreasing }\right\} .
$$

For the properties of $\mathcal{D}_{+}^{\uparrow}$-valued matrices, let us start with a list of some conditions, where $\stackrel{n}{\mathbf{F}}, \mathbf{F}$ are elements of $\mathcal{D}_{+}^{\uparrow[J \times K]}$, and $\stackrel{n}{\mathbf{F}}, \widetilde{\mathbf{F}}$ are their transforms (in $\mathcal{A}_{+}^{[J \times K]}$ ). Moreover, lim stands for the matrix-weak limit as $n \rightarrow \infty$, and $\sum_{n}$ stands for the matrix-weak limit of the partial sums of the series.

(23) Conditions A.

- $\lim \stackrel{n}{\mathbf{F}}(t)=\mathbf{0}_{J, K}$ for all $t \in \mathbb{R}_{+}$;

- $\left(\lim { }^{n} \mathbf{F}(t)^{K} \cdot \mathbf{I}\right)_{j,}=0$ for all $t \in \mathbb{R}_{+}$and $j \in J$;

- $\lim \mathbf{A} \stackrel{J}{*} \stackrel{n}{\mathbf{F}}{ }^{K} \underset{K}{*} \mathbf{B}(t)=0$ for all $t \in \mathbb{R}_{+}, \mathbf{A} \in l^{1}\left(J, \mathcal{D}_{+}\right)$and $\mathbf{B} \in$ $l^{\infty}\left(K, \mathcal{D}_{+}\right)$.

(24) Conditions B.

- $\lim \stackrel{n}{\mathbf{F}}=\mathbf{0}_{J, K}$;

- $\left(\lim { }^{n} \mathbf{F}(\infty)^{K} \cdot \mathbf{I}\right)_{j},=0$ for all $j \in J$;

- $\lim \mathbf{A} * \stackrel{n}{F}^{\mathbf{F}} * \mathbf{K}=0$ for all $\mathbf{A} \in l^{1}\left(J, \mathcal{D}_{+}\right)$and $\mathbf{B} \in l^{\infty}\left(K, \mathcal{D}_{+}\right)$;

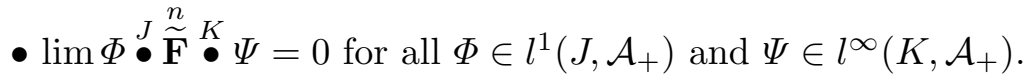

(25) Conditions C.

- $\sum_{n} \stackrel{n}{\mathbf{F}}=\mathbf{F}$;

- $\left(\sum_{n}{ }^{n} \mathbf{F}(\infty)^{K} \cdot \mathbf{I}\right)_{j,}=\left(\mathbf{F}(\infty)^{K} \cdot \mathbf{I}\right)_{j}$, for all $j \in J$;

- $\sum_{n} \mathbf{A} * \stackrel{J}{*} \mathbf{F}^{K} * \mathbf{B}=\mathbf{A} * \mathbf{J} *{ }_{*}^{K} \mathbf{B}$ for all $\mathbf{A} \in l^{1}\left(J, \mathcal{D}_{+}\right)$ and $\mathbf{B} \in l^{\infty}\left(K, \mathcal{D}_{+}\right)$

- $\sum_{n} \Phi(s) \stackrel{J}{\stackrel{n}{\mathbf{F}}(s)} \stackrel{K}{\cdot} \Psi(s)=\Phi(s)^{J} \cdot \widetilde{\mathbf{F}}(s)^{K} \cdot \Psi(s)$ for all $s \in \mathbb{C}_{+}$and for all $\Phi \in l^{1}\left(J, \mathcal{A}_{+}\right)$and $\Psi \in l^{\infty}\left(K, \mathcal{A}_{+}\right)$. 
Proposition 2. For uniformly bounded sequences $\stackrel{n}{\mathbf{F}}$, the statements of each of the above Conditions are mutually equivalent. Moreover, Conditions $\mathrm{C}$ are essentially stronger than Conditions $\mathrm{B}$, and the latter are essentially stronger than Conditions A.

4. The renewal kernels and renewal equations. With the notation of Section 3, we introduce

Definition 2. An $\mathbb{R}_{+}$-valued $(J, K)$-matrix $\mathbf{q}$ is said to be $[s u b]$ stochastic if

$$
\left(\mathbf{q}^{K} \cdot \mathbf{I}\right)_{j,}=1 \quad[\text { resp. } \leq 1] \quad \text { for } j \in J .
$$

Moreover, a $\mathcal{D}_{+}^{\uparrow}$-valued matrix $\mathbf{Q}$ is said to be a $[$ sub]renewal kernel (on $J \times K)$ if $\mathbf{Q}(\infty)$ is [sub] stochastic.

The set of all stochastic [substochastic] matrices is denoted by $p(J, K)$ $[q(J, K)$, respectively]. The set of all renewal [subrenewal] kernels is denoted by $\mathcal{P}(J, K)[\mathcal{Q}(J, K)$, respectively]. Correspondingly, $\mathcal{P}$ [resp. $\mathcal{Q}]$ denotes the family of all [sub-]p.d.f.'s concentrated on $\mathbb{R}_{+}$.

Proposition 3. (i) $p(J, K) \subset q(J, K) \subset \mathbb{R}_{+}^{[J \times K]}$ and $\mathcal{P}(J, K) \subset \mathcal{Q}(J, K)$ $\subset \mathcal{D}_{+}^{\uparrow[J \times K]}$.

(ii) For every $[$ sub] stochastic matrix $\mathbf{q} \in p(J, K)[$ resp. $\mathbf{q} \in q(J, K)]$, we have

- $\|\mathbf{q}\|=\left\|\mathbf{q}^{K} \cdot \mathbf{I}\right\|=1[$ resp. $\leq 1]$

- every submatrix of $\mathbf{q}$ is substochastic.

(iii) For every $[$ sub] renewal kernel $\mathbf{Q} \in \mathcal{P}(J, K)[$ resp. $\mathbf{Q} \in \mathcal{Q}(J, K)]$, we have

- $\mathbf{Q}(t) \in q(J, K)$ for $t \in \mathbb{R}$ and $\widetilde{\mathbf{Q}}(s) \in q(J, K)$ for $s \in \mathbb{R}_{+}$,

- $\left.\|\mathbf{Q}\|_{\text {var }}=\|\mathbf{Q}(\infty)\|=\| \mathbf{Q}(\infty)\right)^{K} \mathbf{I}\|=\| \widetilde{\mathbf{Q}}(0) \|=1[$ resp. $\leq 1]$,

- every submatrix of $\mathbf{Q}$ is a subrenewal kernel.

(iv) The matrix product of two [sub] stochastic matrices is [sub] stochastic.

(v) The matrix convolution of two [sub]renewal kernels is [sub] renewal.

The matrices of transition moment's d.f.'s and the matrices of transition moments of degree $l \in \mathbb{R}_{+}$of the renewal kernel $\mathbf{Q}$ are given by (cf. (10))

$$
\mathbf{Q}^{(l)}:=\left[Q_{j, k}^{(l)} ;(j, k) \in J \times K\right], \quad \mathbf{q}^{(l)}:=\mathbf{Q}^{(l)}(\infty) \subset \overline{\mathbb{R}}_{+}=\mathbb{R}_{+} \cup\{\infty\} .
$$

Then $\mathbf{q}^{(0)}=\mathbf{Q}(\infty) \in p(J, K)$ consists of transition probabilities of $\mathbf{Q}$. The column

$$
\mathbf{G}=\mathbf{Q}^{K} \cdot \mathbf{I}=\left[\sum_{k \in K} Q_{j, k} ; j \in J\right] \in \mathcal{P}(J, \sharp)
$$


consists of unconditional p.d.f.'s assigned to $\mathbf{Q}$. The symbols $\mathbf{G}^{(l)}$ and $\mathbf{g}^{(l)}:=\mathbf{G}^{(l)}(\infty)$ stand for the corresponding $J$-columns of unconditional moment's d.f.'s and unconditional moments, respectively.

Sometimes, we also need the matrix $\mathbf{F}$ of conditional transition p.d.f.'s related to $\mathbf{Q}$ by

$$
Q_{j, k}=q_{j, k} F_{j, k} \quad \text { for }(j, k) \in J \times K, \mathbf{F} \in \mathcal{P}^{J \times K} .
$$

Till the end of this section, $J$ is fixed and we assume that

$$
\mathbf{Q} \in \mathcal{Q}(J, J), \quad \mathbf{1}=\mathbf{1}_{J, J}, \quad \mathbf{I}=\mathbf{I}_{J,}, \quad \mathbf{0}=\mathbf{0}_{J,} .
$$

Also, we omit the prefix " $m w$-" indicating matrix-weak convergence.

In the main part of this work, some probabilities depending on "time" $t$, if collected in a column, say

$$
\mathbf{U}=\left[U_{j}(t) ; j \in J\right]^{\prime} \in \mathcal{D}_{+}^{J \times \sharp},
$$

are shown to form a bounded solution of the renewal equation

$$
\mathbf{U}=\mathbf{Q} * \mathbf{J} \mathbf{U}+\mathbf{V}, \quad \text { where } \mathbf{V} \in \mathcal{D}_{+}^{[J \times \sharp]} .
$$

Proposition 4 (a uniqueness theorem for the renewal equation). The renewal equation (30) has at most one bounded solution $\mathbf{U} \in \mathcal{D}_{+}^{[J \times \sharp]}$ whenever $\mathbf{Q}^{* n}(t)$ converges to $\mathbf{0}_{J, J}$ for $t \in \mathbb{R}_{+}$.

Proof. The difference $\mathbf{W} \in \mathcal{D}_{+}^{[J \times J]}$ of any two bounded solutions of (30) satisfies $\mathbf{W}(t)=\mathbf{Q}^{* m} \stackrel{J}{*} \mathbf{W}(t)$ for $t \in \mathbb{R}_{+}$and $m \in \mathbb{N}$. The equivalence of Conditions A (cf. Proposition 2) implies that $\mathbf{W}(t)=\lim _{m \rightarrow \infty} \mathbf{Q}^{* m_{*}}{ }_{*}^{J} \mathbf{W}(t)=$ $\mathbf{0}_{J}$.

In order to describe the solutions of (30), we use the Neumann series for inverse matrices. By monotonicity arguments, the case of $\mathcal{D}_{+}^{\uparrow}$-valued matrices reduces to $\mathbb{R}_{+}$-valued matrices. The essential fact is

Lemma 1. For every $\mathbf{q} \in q(J, J)$,

$$
\mathbf{s}:=\sum_{m=0}^{\infty} \mathbf{q}^{\cdot m} \in \mathbb{R}^{[J \times J]} \quad \text { iff } \quad \mathbf{v}:=(\mathbf{1}-\mathbf{q})^{-1} \in \mathbb{R}^{[J \times J]} .
$$

In that case, $\mathbf{s}=\mathbf{v}$.

Proof. According to Proposition 2 applied to ${ }^{n} \mathbf{F}(t)=\mathbf{q}^{\cdot n}$, where $n \in$ $\mathbb{N}$ and $t \in \mathbb{R}_{+}, \mathbf{q}^{\cdot n}$ converges to $\mathbf{0}_{J, J}$ whenever the series is convergent. Therefore

$$
\mathbf{s} \cdot(\mathbf{1}-\mathbf{q})=(\mathbf{1}-\mathbf{q}) \cdot \mathbf{s}=\sum_{m=0}^{\infty} \mathbf{q}^{\cdot m} \cdot(\mathbf{1}-\mathbf{q})=\lim _{n \rightarrow \infty}\left(\mathbf{1}-\mathbf{q}^{\cdot n}\right)=\mathbf{1}
$$


On the other hand, for bounded $\mathbf{v}$, we have

$$
\sum_{m=0}^{n-1} \mathbf{q}^{\cdot m}=\mathbf{s}_{n-1}=\mathbf{s}_{n-1} \cdot(\mathbf{1}-\mathbf{q}) \cdot \mathbf{v}=\left(\mathbf{1}-\mathbf{q}^{\cdot n}\right) \cdot \mathbf{v}
$$

Therefore, $\left\|\mathbf{s}_{n-1}\right\| \leq 2\|\mathbf{v}\|$ and the limits of the elements $s_{n ; j, k}$ of $\mathbf{s}_{n}$ form a bounded matrix. Again by Proposition 2, the first condition and the equality $\mathbf{s}=\mathbf{v}$ follow.

In a similar way, one can prove that

LEMMA 2. If the series

$$
\mathbf{U}=\sum_{m=0}^{\infty} \mathbf{Q}^{* m} \underset{*}{J}
$$

is convergent in $\mathcal{D}_{+}^{[J \times \sharp]}$, then $\mathbf{U}$ is a bounded solution of (30).

Combining Lemmas 1 and 2 with Propositions 2 and 4, we come to the following

COROllary 1. Let the subrenewal kernel $\mathbf{Q} \in \mathcal{Q}(J, J)$ satisfy

$$
(\mathbf{1}-\mathbf{Q}(\infty))^{-1} \in \mathbb{R}^{[J \times J]} .
$$

Then the sufficient condition of Proposition 4 is satisfied. Moreover, for every $\mathbf{V}, \mathbf{U} \in \mathcal{D}_{+}^{[J \times \sharp]}$, formula (31) and equation (30) are equivalent and imply that

$$
\widetilde{\mathbf{U}}(a)=(\mathbf{1}-\widetilde{\mathbf{Q}}(a))^{-1} \cdot \widetilde{\mathbf{V}}(a)=\sum_{m=0}^{\infty}(\widetilde{\mathbf{Q}}(a))^{m} \cdot \widetilde{\mathbf{V}}(a) \quad \text { for } a \in \mathbb{R}_{+} \cdot
$$

For the evaluation or estimation of the series of Corollary 1 we follow Korolyuk and Turbin [6]. We consider perturbed stochastic matrices, say,

$$
\mathbf{p} \in p(J, J) \text {. }
$$

Let us assume that the transition probability matrix $\mathbf{p}$ of a Markov chain on $J$ satisfies

$$
\begin{gathered}
\text { some } \boldsymbol{\pi} \in p(\sharp, J) \text { satisfies: } \boldsymbol{\pi}^{J} \cdot \mathbf{I}=1, \quad \boldsymbol{\pi}^{J} \cdot \mathbf{p}=\boldsymbol{\pi} \text { and } \\
\boldsymbol{\Lambda}=(\mathbf{1}-\mathbf{p}+\mathbf{\Pi})^{-1} \in \mathbb{R}_{+}^{[J \times J]}, \quad \text { i.e. } \quad \lambda:=\|\boldsymbol{\Lambda}\|<\infty,
\end{gathered}
$$

where $\boldsymbol{\Pi}:=\mathbf{I} \cdot \boldsymbol{\pi} \in p(J, J)$. Then the chain is said to be strongly recurrent. In particular, there is exactly one stationary p.d. of such a chain and it is equal to $\pi$. For these facts and the following lemma, see [6] and [11].

LEMMA 3 (a perturbation theorem for stochastic matrices). In terms of (32)-(34), let h satisfy

$$
\mathbf{h} \in \mathbb{R}^{[J \times J]} \quad \text { and } \quad \chi:=\boldsymbol{\pi}^{J} \cdot \mathbf{h}^{J} \cdot \mathbf{I} \neq 0,
$$




$$
r:=\lambda\left(1+\frac{\left\|\boldsymbol{\pi}^{J} \cdot \mathbf{h}\right\|}{|\chi|}\right)\left(1+\frac{\left\|\mathbf{h}^{J} \cdot \mathbf{I}\right\|}{|\chi|}\right)\|\mathbf{h}\|<1 .
$$

Then the inverse matrix of $\mathbf{1}-\mathbf{p}+\mathbf{h}$ exists in $\mathbb{R}^{[J \times J]}$ and it is given by

$$
(\mathbf{1}-\mathbf{p}+\mathbf{h})^{-1}=\chi^{-1} \cdot \mathbf{\Pi}+\left(\mathbf{1}+\mathbf{T}^{J} \cdot \mathbf{h}\right)^{-1} \cdot \mathbf{T},
$$

where

$$
\mathbf{T}:=\mathbf{A} \cdot \mathbf{\Lambda}^{J} \cdot \mathbf{B}, \quad \mathbf{A}:=\mathbf{1}-\chi^{-1} \mathbf{\Pi}^{J} \cdot \mathbf{h}, \quad \mathbf{B}:=\mathbf{1}-\chi^{-1} \cdot \mathbf{h}^{J} \cdot \mathbf{\Pi} .
$$

Moreover,

$$
\left\|\left(\mathbf{1}+\mathbf{T}^{J} \cdot \mathbf{h}\right)^{-1} \cdot \mathbf{T}\right\| \leq r(1-r)^{-1}\|\mathbf{h}\|^{-1} .
$$

5. Semi-Markov processes. Let $K$ be an at most countable state space and assume that

$$
\begin{aligned}
\mathbf{p}^{(0)} & =\left[p_{j}^{(0)} ; j \in K\right] \in p(K, K), \\
\mathbf{Q} & =\left[Q_{j, k} ;(j, k) \in K \times K\right] \in \mathcal{P}(K, K) .
\end{aligned}
$$

The renewal kernel $\mathbf{Q}$ and the p.d. $\mathbf{p}^{(0)}$ allow us to construct a homogeneous two-dimensional Markov chain

$$
(\vartheta, \xi):=\left(\left(\vartheta_{m}, \xi_{m}\right) ; m \in \mathbb{N}\right) \in\left(\mathbb{R}_{+} \times K\right)^{\mathbb{N}} \quad \text { w.p. } 1,
$$

satisfying the following equalities for $m \in \mathbb{N}, u \in \mathbb{R}$ and $k \in K$ :

(37) $\operatorname{Pr}\left\{\vartheta_{m+1} \leq u, \xi_{m+1}=k \mid \vartheta_{m}, \xi_{m}\right\}=Q_{\xi_{m}, k}(u) \quad$ w.p. 1, and

$$
\operatorname{Pr}\left\{\vartheta_{0}=0, \xi_{0}=j\right\}=p_{j}^{(0)}, \quad j \in K \text {. }
$$

As a direct consequence of the defining conditions (26)-(28) and (37), we get

(38) $\operatorname{Pr}\left\{\xi_{0}=j_{0}, \xi_{l}=j_{l}\right.$ and $\vartheta_{l} \leq u_{l}$ for $\left.l=1, \ldots, m\right\}$

$$
=p_{j_{0}}^{(0)} \prod_{l=1}^{m} Q_{j_{l-1}, j_{l}}\left(u_{l}\right)=p_{j_{0}}^{(0)} \prod_{l=1}^{m} q_{j_{l-1}, j_{l}} F_{j_{l-1}, j_{l}}\left(u_{l}\right) .
$$

Then we define w.p. 1

$$
\begin{gathered}
(\tau, \xi):=\left(\left(\tau_{m}, \xi_{m}\right) ; m \in \mathbb{N}\right): \Omega \rightarrow\left(\mathbb{R}_{+} \times K\right)^{\mathbb{N}}, \quad \text { where } \\
\tau_{m}=\sum_{l=1}^{m} \vartheta_{l} \quad \text { for } m \in \mathbb{N}, \quad \tau_{\infty}=\lim _{m \rightarrow \infty} \tau_{m}=\sup \left\{\tau_{m}: m \in \mathbb{N}\right\},
\end{gathered}
$$

and

(40) $X(\cdot):=\left(X(t)=\xi_{\nu(t)} ; 0 \leq t<\tau_{\infty}\right), \quad$ where $\quad \nu(t):=\sum_{m=1}^{\infty} \chi_{[0, t]}\left(\tau_{m}\right)$. 
Definition 3. The sequence $(\tau, \xi)$ given by (39) is called a Markov renewal chain on $K$ with the initial p.d. of $\xi$ equal to $\mathbf{p}^{(0)}$, related to the renewal kernel $\mathbf{Q}$. The r.v.'s $\tau_{m}, \xi_{m}$ and $\vartheta_{m}$ are called the instant, the state of the $m$ th renewal and the distance between $m$ th consecutive renewals, respectively.

The process $X(\cdot)=\left(X(t) ; t \in\left[0, \tau_{\infty}\right)\right)$ with random duration $\tau_{\infty}$ given by (39)-(40) is called a homogeneous semi-Markov process (HSMP) on $K$, generated by the Markov renewal chain $(\tau, \xi)$ related to the renewal kernel $\mathbf{Q}$.

The name of $(\tau, \xi)$ is justified by the first part of the following list of standard properties of HSMP.

Proposition 5. (i) $(\tau, \xi)$ is a Markov chain and for $m \in \mathbb{N}, u \in \mathbb{R}_{+}$and $k \in K$, the following holds w.p. 1 :

- $\operatorname{Pr}\left\{\tau_{m+1} \leq u, \xi_{m+1}=k \mid \tau_{m}, \xi_{m}\right\}=Q_{\xi_{m}, k}\left(u-\tau_{m}\right)$,

- $\tau_{0}=0 \leq \tau_{1} \leq \tau_{2} \leq \ldots$,

- $\nu(t)=m$ iff $\tau_{m} \leq t<\tau_{m+1}$, and $\tau_{\infty}>t$ iff $\nu(t)<\infty$,

- the sample paths of $X(\cdot)$ are right-continuous and constant between the instants of the renewals,

- the points of discontinuity $\sigma_{l}, l \in \mathbb{N}, \sigma_{0}:=0$, of $X(\cdot)$ form a (finite or infinite) subsequence of $\left\{\tau_{0}, \tau_{1}, \tau_{2}, \ldots\right\}$.

(ii) If additionally $\mathbf{Q}(0)=\mathbf{0}_{K, K}$ (each entry of $\mathbf{Q}$ is continuous at 0 ), then we also have:

- $\tau$ is strictly increasing (and tends to $\tau_{\infty} \leq \infty$ as $n \rightarrow \infty$ ),

- $X\left(\tau_{m}\right)=\xi_{m}$,

- $\left\{\sigma_{0}, \sigma_{1}, \sigma_{2}, \ldots\right\}=\left\{\tau_{0}, \tau_{1}, \tau_{2}, \ldots\right\}$ whenever $q_{k, k}=0$ for $k \in K$.

Corollary 2. (i) $\xi=\left(\xi_{m} ; m \in \mathbb{N}\right): \Omega \rightarrow K^{\mathbb{N}}$ is a Markov chain with $\mathbf{p}^{(0)}$ as initial p.d. and with the stochastic matrix $\mathbf{q}$ as transition probability matrix; in particular,

$$
\operatorname{Pr}\left\{\xi_{m+1}=j \mid \xi_{0}, \xi_{1}, \ldots, \xi_{m-1}, \xi_{m}\right\}=q_{\xi_{m}, j} .
$$

(ii) The distances $\vartheta=\left(\vartheta_{m} ; m \in \mathbb{N}\right)$ are conditionally independent with respect to the $\sigma$-field generated by $\xi$ in the following sense:

$$
\begin{aligned}
\operatorname{Pr}\left\{\vartheta_{1} \leq u_{1}, \ldots, \vartheta_{m+1}\right. & \left.\leq u_{m+1} \mid \xi_{0}, \xi_{1}, \ldots, \xi_{M-1}, \xi_{M}\right\} \\
& = \begin{cases}\prod_{l=1}^{m} F_{\xi_{l-1}, \xi_{l}}\left(u_{l}\right) G_{\xi_{m}}\left(u_{m+1}\right) & \text { if } M=m, \\
\prod_{l=1}^{m+1} F_{\xi_{l-1}, \xi_{l}}\left(u_{l}\right) & \text { if } M>m .\end{cases}
\end{aligned}
$$

(iii) For $m \in \mathbb{N}, t \in \mathbb{R}_{+}$and $J_{0}, J_{1}, \ldots, J_{m} \subset K$ we also have

$\operatorname{Pr}\left\{\tau_{m} \leq t\right.$ and $\xi_{l} \in J_{l}$ for $\left.l=0,1, \ldots, m\right\}$

$$
=\mathbf{p}^{(0)} \stackrel{J_{0}}{\cdot} \mathbf{Q} \stackrel{J_{1}}{*} \mathbf{Q} \stackrel{J_{2}}{*} \ldots{ }^{J_{m-1}}{ }^{*} \mathbf{Q}(t) \stackrel{J_{m}}{\cdot} \mathbf{I}
$$




$$
\begin{aligned}
\operatorname{Pr}\left\{\tau_{m} \leq t<\tau_{m+1} \text { and } \xi_{l}\right. & \left.\in J_{l} \text { for } l=0,1, \ldots, m\right\} \\
& =\mathbf{p}^{(0)}{\stackrel{J_{0}}{\cdot}}^{\mathbf{Q}} \stackrel{J_{1}}{*} \mathbf{Q} \stackrel{J_{2}}{*}{ }^{J_{m-1}}{ }^{*} \mathbf{Q}{ }^{J_{m}}(\mathbf{I}-\mathbf{G})(t) .
\end{aligned}
$$

(iv) If $\mathbf{Q}(0)=\mathbf{0}_{K, K}$, then the equalities of (i)-(iii) remain valid with $\xi_{l}$ replaced by $X\left(\tau_{l}\right), l \in \mathbb{N}$.

Remark 2. The central equality of (ii) of Proposition 5 justifies the name of the embedded Markov chain used for $\xi_{m}=X\left(\tau_{m}\right), m \in \mathbb{N}$. But even if $\tau$ is not strictly increasing, the above equality with $\sigma$ substituted for $\tau$ defines a Markov chain, possibly related to another renewal kernel. This implies non-uniqueness of the renewal kernel corresponding to a given HSMP.

6. The first exit from a subset. We keep the assumptions and notation (35)-(40). In order to avoid some unnecessary complexity, we assume that

$$
\mathbf{Q}(0)=\mathbf{0}_{K, K} .
$$

Then the instant of the first exit of the HSMP $X(\cdot)$ from $J, J \subset K$, is defined by

$$
T_{J}:= \begin{cases}0 & \text { if } X(0) \in K \backslash J, \\ \sup \left\{t: t<\tau_{\infty}, X(u) \in J \text { for } u \leq t\right\} & \text { if } X(0) \in J .\end{cases}
$$

According to Proposition 5 and Corollary 2,

$$
T_{J}=\tau_{\nu_{J}},
$$

where

$$
\nu_{J}= \begin{cases}0 & \text { if } \xi_{0} \in K \backslash J, \\ \sup \left\{n \in \mathbb{N}: \xi_{l} \in J \text { for } l=0,1, \ldots, n-1\right\} & \text { if } \xi_{0} \in J .\end{cases}
$$

Before we evaluate the sub-p.d.f. of $T_{J}$, i.e.

$$
F_{T_{J}}:=\left(F_{T_{J}}(t):=\operatorname{Pr}\left\{T_{J} \leq t\right\} ; t \in \mathbb{R}_{+}\right) \in \mathcal{Q},
$$

let us compare it to

(45) $\quad F_{T_{J}<\tau_{\infty}}=\left(F_{T_{J}<\tau_{\infty}}(t):=\operatorname{Pr}\left\{T_{J} \leq t, T_{J}<\tau_{\infty}\right\} ; t \in \mathbb{R}_{+}\right) \leq F_{T_{J}}$.

Proposition 6. The following conditions are equivalent:

$$
\begin{gathered}
\nu_{J}<\infty \quad \text { w.p. } 1 ; \\
T_{J}<\tau_{\infty} \quad \text { w.p. } 1 ; \\
F_{T_{J}<\tau_{\infty}}=F_{T_{J}} ; \\
F_{T_{J}<\tau_{\infty}}(\infty):=\lim _{t \rightarrow \infty} F_{T_{J}<\tau_{\infty}}(t)=1 .
\end{gathered}
$$

Proof. The implications (i) $\Leftrightarrow$ (ii) $\Leftrightarrow($ iii $) \Rightarrow$ (iv) are direct consequences of (41)-(45) and the finiteness of all instants $\tau_{0}<\tau_{1}<\tau_{2}<\ldots$ (cf. 
Proposition 5). If (iv) holds, then $\operatorname{Pr}\left\{\nu_{J}=\infty\right\}$ is not greater than $\operatorname{Pr}\left\{\nu_{J}=\infty\right.$ or $\left.T_{J}>t\right\}=1-F_{T_{J}<\tau_{\infty}}(t) \rightarrow 0$ as $t \rightarrow \infty$.

By the definition of $T_{J}$ and Corollary 2, for $t \in \mathbb{R}_{+}, F_{T_{J}<\tau_{\infty}}(t)$ equals

(46) $\operatorname{Pr}\left\{\xi_{0} \in K \backslash J\right\}+\sum_{m=0}^{\infty} \operatorname{Pr}\left\{\tau_{m+1} \leq t, \xi_{0}, \ldots, \xi_{m} \in J, \xi_{m+1} \in K \backslash J\right\}$

$$
=\mathbf{p}^{(0)} \stackrel{K \backslash J}{\cdot} \mathbf{I}+\mathbf{p}^{(0)} \cdot \mathbf{F}_{J}(t),
$$

where

$$
\mathbf{F}_{J,}:=\sum_{m=0}^{\infty}\left(\left(\mathbf{Q}_{J, J}\right)^{* m} * \mathbf{Q}_{J, K \backslash J} \cdot \mathbf{I}_{K \backslash J}\right) \in \mathcal{Q}(J,) .
$$

Moreover, for $t \in \mathbb{R}_{+} \cup\{\infty\}$ we have

(48) $\quad F_{T_{J}}(t)-F_{T_{J}<\tau_{\infty}}(t)=\operatorname{Pr}\left\{\tau_{\infty} \leq t, \nu_{J}=\infty\right\}$

$$
=\lim _{m \rightarrow \infty} \operatorname{Pr}\left\{\tau_{m} \leq t, \nu_{J}>m\right\}=\lim _{m \rightarrow \infty} \mathbf{p}^{(0)}{ }^{J} \cdot\left(\mathbf{Q}_{J, J}\right)^{* m}(t)^{J} \cdot \mathbf{I} .
$$

In particular, taking $t=\infty$ in the last two equalites, we get

$$
\operatorname{Pr}\left\{\nu_{J}=\infty\right\}=\lim _{m \rightarrow \infty} \mathbf{p}^{(0)} \stackrel{J}{\cdot}\left(\mathbf{q}_{J, J}\right)^{\cdot m} \cdot \mathbf{I} .
$$

Note that Corollary 2 implies

Proposition 7.

(i) $\operatorname{Pr}\left\{X(t) \in J, \tau_{\infty}>t\right\}=\sum_{m=0}^{\infty} \operatorname{Pr}\left\{\tau_{m} \leq t<\tau_{m+1}, \xi_{m} \in J\right\}$

$$
=\mathbf{p}^{(0)} \stackrel{K}{\sum_{m=0}^{\infty}} \mathbf{Q}^{* m}{ }_{*}^{J}(\mathbf{I}-\mathbf{G})(t),
$$

(iii) $\quad \operatorname{Pr}\left\{\tau_{\infty}=\infty\right\}=1-\lim _{t \rightarrow \infty} \lim _{m \rightarrow \infty} \mathbf{p}^{(0)}{ }^{K} \cdot \mathbf{Q}^{* m}(t)^{K} \cdot \mathbf{I}$,

(iv) $\operatorname{Pr}\left\{\tau_{\infty}=\infty, \nu_{J}=\infty\right\}$

$$
=\operatorname{Pr}\left\{\nu_{J}=\infty\right\}-\lim _{t \rightarrow \infty} \lim _{m \rightarrow \infty} \mathbf{p}^{(0)}{ }^{J} \cdot\left(\mathbf{Q}_{J, J}\right)^{* m}(t)^{J} \cdot \mathbf{I} .
$$

Corollary 3. The J-column $\mathbf{F}_{J}$, given by (47) satisfies the renewal equation

$$
\mathbf{F}_{J,}=\mathbf{Q}_{J, J} * \mathbf{F}_{J,}+\mathbf{Q}_{J, K \backslash J} * \mathbf{I}_{K \backslash J,} .
$$

If additionally $\mathbf{1}_{J, J}-\mathbf{q}_{J, J}$ with $\mathbf{q}_{J, J}=\mathbf{Q}_{J, J}(\infty)$ is invertible in $\mathbb{R}_{+}^{[J \times J]}$, then (ii) $\quad \widetilde{\mathbf{F}}_{J}(s)=\left(\mathbf{1}_{J, J}-\widetilde{\mathbf{Q}}_{J, J}(s)\right)^{-1} \cdot \widetilde{\mathbf{Q}}_{J, K \backslash J}(s) \cdot \mathbf{I}_{K \backslash J}, \quad$ for $s \in \mathbb{C}_{+}$, 
where

(iii) $\quad F_{T_{J}}=F_{T_{J}<\tau_{\infty}}=\mathbf{p}^{(0)}{ }^{J} \cdot \mathbf{F}_{J} \in \mathcal{P} \quad$ whenever $X(0) \in J$ w.p. 1.

Proof. For (i) and (ii), use Corollary 1 of Section 4. According to it and (49), $\nu_{J}<\infty$ w.p. 1. Therefore, Proposition 6 is applicable, leading to the first equality in (iii) for every initial p.d. (of $\xi_{0}$ ). For processes starting in $J,(46)$ gives us the second equality in (iii).

Remark 3. The p.d. of the time to the first exit from a subset $J$ depends on the renewal kernel through the submatrices $\mathbf{Q}_{J, J}$ and $\mathbf{Q}_{J, \sharp}:=$ $\mathbf{Q}_{J, K \backslash J} \cdot \mathbf{I}_{K \backslash J}$, only. In other words, the time $T_{J}$ to the first exit will have the same p.d. if $K$ and $\mathbf{Q}_{J, K \backslash J}$ are replaced by $J \cup\{\sharp\}$ and $\mathbf{Q}_{J, \sharp}$, respectively. Moreover, one can assume without loss that the states of $K \backslash J$ which are represented by $\sharp$ are all absorbing.

7. The limit theorem for the first exit. Our goal is to analyse the limit p.d. of the first exit times for a sequence $X^{n}(\cdot), n \in \mathbb{N}$, of HSMProcesses on a common at most countable state space $K$. They all have the same initial p.d. concentrated on a subset, say $J$, of the state space, not equal to $K$. Therefore without loss we assume that (cf. Remark 3 in Section 6)

$$
J \neq \emptyset, \quad J \text { is at most countable, } \quad K=J \cup\{\sharp\} .
$$

The common initial p.d. is described by

$$
\mathbf{p}^{(0)}:=[\operatorname{Pr}\{\stackrel{n}{X}(0)=j ; j \in K\}] \in p(\sharp, K), \quad \text { where } \mathbf{p}^{(0)} \cdot \mathbf{I}=1 .
$$

For $n \in \mathbb{N}$, let the process $\stackrel{n}{X}(\cdot)$ be related to the renewal kernel

$$
\stackrel{n}{\mathbf{Q}} \in \mathcal{P}(K, K), \quad \text { where } \quad \stackrel{n}{\mathbf{Q}}(0)=\mathbf{0}_{K, K}, \quad \stackrel{n}{\mathbf{Q}_{\sharp}, J}(\infty)=\mathbf{0}_{\sharp, J} .
$$

The d.f. of our interest is then defined by the equalities (cf. Section 6)

$$
\begin{aligned}
& \stackrel{n}{F}_{J}(t):=\operatorname{Pr}\left\{\stackrel{n}{T}_{J} \leq t\right\}, \quad t \in \mathbb{R}_{+}, \quad \text { where } \\
& \stackrel{n}{T}_{J}=\sup \left\{t: t<\tau_{\infty}, \stackrel{n}{X}(u) \in J \text { for } u \leq t\right\} .
\end{aligned}
$$

Theorem 2 below concerns sequences for which the central submatrices $\stackrel{n}{\mathbf{Q}}_{J, J}$ tend to a renewal kernel

(54) $\quad \mathbf{P} \in \mathcal{P}(J, J)$, where $\mathbf{p}=\mathbf{P}(\infty) \in p(J, J)$ corresponds to a strongly recurrent Markov chain.

Thus, we can introduce the (unique) stationary p.d. $\pi$ of the matrix $\mathbf{p}$, the matrices $\boldsymbol{\Lambda}, \boldsymbol{\Pi}$ and the number $\lambda$ according to (33)-(34). 
The assumptions on the perturbation are expressed in terms of the stationary average p.d.f. of the distances in the limit HSMP

$$
F_{\vartheta}:=\pi^{J} \cdot \mathbf{P}^{J} \cdot \mathbf{I} \in \mathcal{P},
$$

and the differences of transformed renewal kernels and their averages given by the following equalities:

$$
\begin{aligned}
& \mathbf{H}_{\vartheta}:=\mathbf{p}-\widetilde{\mathbf{P}} \in \mathcal{A}_{+}^{[J \times J]}, \quad H_{\vartheta}:=\pi^{J} \cdot \mathbf{H}_{\vartheta}{ }^{J} \cdot \mathbf{I}=1-\widetilde{F}_{\vartheta} \in \mathcal{A}_{+}, \\
& {\stackrel{n}{\boldsymbol{\Delta}_{\text {in }}}}:=\widetilde{\mathbf{P}}-\stackrel{n}{\mathbf{Q}}_{J, J} \in \mathcal{A}_{+}^{[J \times J]}, \quad{\stackrel{n}{\mathrm{in}}}^{[J}=\boldsymbol{\pi} \cdot{\stackrel{n}{\boldsymbol{\Delta}_{\text {in }}}}^{J} \cdot \mathbf{I} \in \mathcal{A}_{+}, \\
& {\stackrel{n}{\boldsymbol{\Delta}_{\text {out }}}}:=\stackrel{n}{\mathbf{Q}}_{J, \sharp} \in \mathcal{A}_{+}^{[J \times \sharp]}, \quad \stackrel{n}{\Delta}_{\text {out }}:=\pi{ }^{J}{\stackrel{n}{\Delta_{\text {out }}}}^{J} \cdot \mathbf{I} \in \mathcal{A}_{+}, \\
& \varepsilon_{n}:=\stackrel{n}{\Delta}_{\text {out }}(0) \quad\left(=\stackrel{n}{\Delta}_{\text {in }}(0)\right) .
\end{aligned}
$$

TheOREM 2. Let the HSMProcesses $\stackrel{n}{X}(\cdot)$ on $K$ and the limit renewal kernel $\mathbf{P}=\mathbf{P}_{J, J}$ be given by (50)-(52) and (54), (33)-(34), respectively. In terms of (55)-(59), suppose that the renewal kernels $\stackrel{n}{\mathbf{Q}}$ of $\stackrel{n}{X}(\cdot)$ satisfy the following conditions:

(a) $\quad \varepsilon_{n}>0, \quad \varepsilon_{n} \rightarrow 0, \quad \varepsilon_{n}^{-1} \varepsilon_{n+1} \rightarrow 1, \quad \varepsilon_{n}^{-1} \stackrel{n}{\Delta_{\text {out }}}\left(\varepsilon_{n}\right) \rightarrow 1 \quad$ as $n \rightarrow \infty$;

(b) $\quad \varrho^{2}(a):=\left(H_{\vartheta}(a)\right)^{-2}\left\|\mathbf{H}_{\vartheta}(a)\right\|^{3} \rightarrow 0 \quad$ as $a \rightarrow 0^{+}$.

Moreover, suppose that the perturbation $\stackrel{n}{\Delta}_{\text {in }}$ satisfy

$$
\stackrel{n}{\Delta_{\text {in }}}(a)>0 \quad \text { for } a \in \mathbb{R}_{+},
$$

and that almost uniformly with respect to $a \in \mathbb{R}_{+}$,

$$
\begin{gathered}
\varepsilon_{n}^{-1} \stackrel{n}{\Delta}_{\mathrm{in}}\left(\varepsilon_{n} a\right) \rightarrow 1 \quad \text { as } n \rightarrow \infty, \\
\sigma_{n}^{2}(a):=\left(\stackrel{n}{\Delta}_{\mathrm{in}}(a)\right)^{-2}\left\|\Delta_{\mathrm{in}}(a)\right\|^{3} \rightarrow 0 \quad \text { as } n \rightarrow \infty .
\end{gathered}
$$

(i) The following conditions are equivalent:

- for some constants $c_{n} \rightarrow 0^{+}$, the p.d.f. $\stackrel{n}{F}{ }_{J}$ of $c_{n} \stackrel{n}{T}_{J}$ given by (53) is weakly convergent to a p.d.f. which is not concentrated on $\{0\}$;

- $1-\widetilde{F}_{\vartheta}$ is regularly varying at 0 , with a positive degree.

(ii) Under the conditions of (i), for some constants $c>0$ and $\alpha \in(0,1]$, the transform of the d.f. $\stackrel{n}{F}_{J}$ satisfies

$$
\stackrel{n}{F}_{J}\left(c_{n} s\right) \rightarrow\left(1+c s^{\alpha}\right)^{-1}, \quad s \in \mathbb{C}_{+}, \quad \text { as } n \rightarrow \infty .
$$

Proof. Let us start with an arbitrary sequence $c_{n} \rightarrow 0^{+}$and take $a \geq 0$ 
in the following notation and estimates for elements of $J \times J$-matrices:

$$
\begin{aligned}
\mathbf{h}_{n}(a) & :=\mathbf{p}-\widetilde{\mathbf{Q}}_{J, J}\left(c_{n} a\right)=\mathbf{H}_{\vartheta}\left(c_{n} a\right)+\stackrel{n}{\text { in }}_{\text {in }}\left(c_{n} a\right) \\
& =\stackrel{n}{\text { in }}_{\text {in }}(0)+\stackrel{n}{\mathbf{q}}_{J, J}-\widetilde{\mathbf{Q}}_{J, J}\left(c_{n} a\right) \geq \stackrel{n}{\text { in }}_{\text {in }}(0)=\mathbf{h}_{n}(0),
\end{aligned}
$$

where $\stackrel{n}{\mathbf{q}}:=\stackrel{n}{\mathbf{Q}}(\infty)=\stackrel{n}{\mathbf{Q}}(0)$. From the equalities

$$
\widetilde{n}_{J, K}(0)^{K} \cdot \mathbf{I}=\widetilde{\mathbf{P}}(0)^{J} \cdot \mathbf{I}=\mathbf{I}_{J}, \quad \text { and } \quad H_{\vartheta}=1-\widetilde{F}_{\vartheta}
$$

we get the following estimates for the elements of the $J$-columns:

$$
\begin{aligned}
& \mathbf{h}_{n}(a) \stackrel{J}{J} \mathbf{I} \geq \mathbf{h}_{n}(0)^{J} \cdot \mathbf{I}=\stackrel{n}{\Delta}_{\text {in }}(0)^{J} \cdot \mathbf{I}=\left(\widetilde{\mathbf{P}}(0)-\widetilde{n}_{J, J}(0)\right)^{J} \cdot \mathbf{I}=\widetilde{\mathbf{Q}}_{J, \sharp}(0) \\
& =\stackrel{n}{\Delta}_{\text {out }}(0) \geq \stackrel{n}{\mathbf{Q}}_{J, \sharp}\left(c_{n} a\right)=\stackrel{n}{\Delta}_{\text {out }}\left(c_{n} a\right) .
\end{aligned}
$$

Moreover, the norms of $\mathbf{h}_{n}$ and the numbers

$$
\chi_{n}(a):=\boldsymbol{\pi}^{J} \cdot \mathbf{h}_{n}(a)^{J} \cdot \mathbf{I}
$$

satisfy

$$
\begin{gathered}
2 \geq\left\|\mathbf{h}_{n}(a)\right\| \geq \chi_{n}(a)=1-\widetilde{F}_{\vartheta}\left(c_{n} a\right)+\stackrel{n}{\Delta_{\text {in }}}\left(c_{n} a\right) \\
\geq \stackrel{n}{\Delta}_{\text {in }}(0)=\stackrel{n}{\Delta}_{\text {out }}(0)=\varepsilon_{n} \geq \stackrel{n}{\Delta}_{\text {out }}\left(c_{n} \cdot a\right)>0 .
\end{gathered}
$$

The appropriate estimates for the numbers

$$
0 \leq r_{n}(a):=\lambda\left(\frac{\chi_{n}(a)}{\left\|\mathbf{h}_{n}(a)\right\|}+1\right)^{2} \frac{\left\|\mathbf{h}_{n}(a)\right\|^{3}}{\chi_{n}(a)^{2}}
$$

are based on the following consequence of the Jensen inequality:

$$
\begin{aligned}
\frac{\left\|\mathbf{h}_{n}(a)\right\|^{3}}{\chi_{n}(a)^{2}} & \leq \frac{\left\|\mathbf{H}_{\vartheta}\left(c_{n} a\right)+\Delta_{\text {in }}\left(c_{n} a\right)\right\|^{3}}{\left(\left(1-\widetilde{F}_{\vartheta}\left(c_{n} a\right)\right)+\Delta_{\text {in }}\left(c_{n} a\right)\right)^{2}} \\
& \leq 2\left[\frac{\left\|\mathbf{H}_{\vartheta}\left(c_{n} a\right)\right\|^{3 / 2}+\left\|\boldsymbol{\Delta}_{\text {in }}\left(c_{n} a\right)\right\|^{3 / 2}}{H_{\vartheta}\left(c_{n} a\right)+\Delta_{\text {in }}\left(c_{n} a\right)}\right]^{2} \leq 2\left[\varrho\left(c_{n} a\right)+\sigma_{n}\left(c_{n} a\right)\right]^{2} .
\end{aligned}
$$

Since $c_{n}$ tends to 0 , by (b) and (c.3), $r_{n}(a) \rightarrow 0^{+}$almost uniformly with respect to $a \in \mathbb{R}_{+}$as $n \rightarrow \infty$. Thus, for every $a_{0} \geq 0$ and $n$ sufficiently large, Lemma 3 is applicable with $\chi=\chi_{n}(a), r=r_{n}(a)$ and $\mathbf{h}=\mathbf{h}_{n}(a)$, assuring $r<1$, for all $0 \leq a \leq a_{0}$. Consequently, the matrix

$$
\mathbf{1}_{J, J}-\widetilde{\mathbf{Q}}_{J, J}(a)=\mathbf{1}_{J, J}-\mathbf{p}+\mathbf{h}_{n}(a)
$$

has an inverse in $\mathbb{R}_{+}^{[J \times J]}$ for $0 \leq a \leq a_{0}$ and $n \geq N_{0} \in \mathbb{N}$. Here, $N_{0}$ depends on $a_{0}$ only. In particular, for these naturals, $\mathbf{1}_{J, J}-\stackrel{n}{\mathbf{q}}_{J, J}$ is also invertible, 
since $a=0$ can be taken. According to Corollary 3,

$$
\stackrel{n}{T}_{J}<\tau_{\infty} \leq \infty \quad \text { w.p. } 1 \text { for } n \geq N_{0}
$$

Thus, for the p.d.f. of $c_{n} \stackrel{n}{T}_{J}$ we have

$$
F_{n}(t)=\stackrel{n}{F}_{J}\left(t / c_{n}\right)=\operatorname{Pr}\left\{c_{n} \stackrel{n}{T}_{J} \leq t\right\}=\operatorname{Pr}\left\{c_{n} \stackrel{n}{T}_{J} \leq t, \stackrel{n}{T}_{J}<\tau_{\infty}\right\}
$$

Moreover, keeping a fixed, for the transform of $F_{n}$ we have the formula (cf. Corollary 3, Lemma 3 and the notation (55)-(59))

$$
\begin{aligned}
& \left.\widetilde{F}_{n}(a)=\mathbf{p}^{(0)} \stackrel{J}{\widetilde{\mathbf{F}}_{J}}\left(c_{n} a\right)=\mathbf{p}^{(0)} \stackrel{J}{J}_{(\mathbf{I}}^{J} \cdot \boldsymbol{\pi} \cdot \chi_{n}(a)^{-1}+\mathbf{E}_{n}(a)\right)^{J} \cdot \stackrel{n}{\boldsymbol{\Delta}}_{\text {out }}\left(c_{n} a\right) \\
& =\frac{\Delta_{\text {out }}\left(c_{n} a\right)}{\Delta_{\text {in }}\left(c_{n} a\right)+\left(1-\widetilde{F}_{\vartheta}\left(c_{n} a\right)\right)}+E_{n}(a) .
\end{aligned}
$$

By Lemma 3(ii), the remainders

$\mathbf{E}_{n}(a)=\left(\mathbf{1}_{J, J}+\mathbf{T}_{n}(a) \cdot \mathbf{h}_{n}(a)\right)^{-1} \mathbf{T}_{n}(a)$ and $E_{n}(a)=\mathbf{p} \cdot \mathbf{E}_{n}(a){ }^{J} \cdot \stackrel{\Delta}{\Delta}_{\text {out }}\left(c_{n} a\right)$ are bounded as follows:

$$
\left|E_{n}(a)\right| \leq\left\|\mathbf{E}_{n}(a)\right\| \cdot\left\|\boldsymbol{\Delta}_{\text {out }}\left(c_{n} a\right)\right\| \leq \frac{r_{n}(a)}{1-r_{n}(a)} \cdot \frac{\left\|\boldsymbol{\Delta}_{\text {out }}\left(c_{n} a\right)\right\|}{\left\|\mathbf{h}_{n}\right\|} \leq \frac{r_{n}(a)}{1-r_{n}(a)} .
$$

Thus, for every sequence $c_{n} \rightarrow 0^{+}$, we have $\left|E_{n}(a)\right| \rightarrow 0$ as $n \rightarrow \infty$.

Condition (c.1) and the obtained formula for $\widetilde{F}_{n}(a)$ show that

$$
\limsup _{n \rightarrow \infty} \widetilde{F}_{n}(a) \leq \limsup _{n \rightarrow \infty} \varepsilon_{n}\left(1-\widetilde{F}_{\vartheta}\left(c_{n} a\right)\right)^{-1} .
$$

Therefore, if the first condition of (i) is satisfied with (positive) $c_{n} \rightarrow 0^{+}$, then the sequence $\varepsilon_{n}{ }^{-1}\left(1-\widetilde{F}_{\vartheta}\left(c_{n} a\right)\right)$ is bounded. Otherwise the sequence $\widetilde{F}_{n}(a)$ would have a cluster point at 0 , which is impossible for the transforms of p.d.f. weakly convergent to a p.d.f. By convexity of $\widetilde{F}_{\vartheta}$, the ratio $c_{n} / \varepsilon_{n}$ also remains bounded. Now the conditions (a) and (c.2) show that the limits of $\widetilde{F}_{n}(a)$ and $\varepsilon_{n}^{-1}\left(\varepsilon_{n}+\left(1-\widetilde{F}_{\vartheta}\left(c_{n} a\right)\right)\right)^{-1}$ coincide for every $a \in \mathbb{R}_{+}$, and equal the Laplace-Stieltjes transform of a p.d.f. on $\mathbb{R}_{+}$. Using Theorem 1 we obtain the second condition of (i).

Conversely, if $\widetilde{F}_{\vartheta}$ is regularly varying at 0 with positive degree of regularity $\alpha$, then $\alpha \in(0,1]$, since $\widetilde{F}_{\vartheta}$ is concentrated on $\mathbb{R}_{+}$. Then, taking for $c_{n}$ the only solution of $1-F_{\vartheta}\left(c_{n}\right)=\varepsilon_{n}$, we get the boundedness of $c_{n} / \varepsilon_{n}$ and

$$
\lim _{n \rightarrow \infty} \frac{1-\widetilde{F}_{\vartheta}\left(c_{n} a\right)}{\varepsilon_{n}}=\lim _{n \rightarrow \infty} \frac{1-\widetilde{F}_{\vartheta}\left(c_{n} a\right)}{1-\widetilde{F}_{\vartheta}\left(c_{n}\right)}=a^{\alpha} \quad \text { for } a \in \mathbb{R}_{+} .
$$

Directly from (a), (c.2) and the formula for $\widetilde{F}_{n}(a)$ we obtain the first condition of (i). Moreover, the assertion (ii) follows with $c=1$. Since the shape 
of the proper limit p.d.f. is independent of the normalizing factors, (ii) holds for any other choice of the sequence $\left(c_{n}\right)$ satisfying the first part of (i).

Acknowledgements. The authors thank Professor Zbigniew Ciesielski for valuable discussions. Special thanks are due to the referee who has suggested important comments.

\section{References}

[1] S. Asmussen, Applied Probability and Queues, Wiley, Chichester, 1987.

[2] W. Feller, An Introduction to Probability Theory and Its Applications, Vol. 2, Wiley, New York, 1971.

[3] I. B. Gertsbakh, Asymptotic methods in reliability theory: a review, Adv. Appl. Probab. 16 (1984), 147-175.

[4] J. Keilson, A limit theorem for passage times in ergodic regenerative processes, Ann. Math. Statist. 37 (1966), 866-870.

[5] B. Kopociński, An Outline of Renewal and Reliability Theory, PWN, Warszawa, 1973 (in Polish).

[6] V. S. Korolyuk and A. F. Turbin, Semi-Markov Processes and Their Applications, Naukova Dumka, Kiev, 1976 (in Russian).

[7] E. Seneta, Regularly Varying Functions, Lecture Notes in Math. 508, Springer, Berlin, 1976.

[8] D. S. Silvestrov, Semi-Markov Processes with a Discrete State Space, Sovetskoe Radio, Moscow, 1980 (in Russian).

[9] A. D. Solovyev, Asymptotic behavior of the time of the first occurrence of a rare event, Engnrg. Cybernetics 9 (1971), 1038-1048.

[10] —, Analytical Methods of the Reliability Theory, WNT, Warszawa, 1979 (in Polish).

[11] A. F. Turbin, Applications of the inversion of linear operators perturbed on the spectrum to some asymptotic problems connected with Markov chains and semiMarkov processes, Teor. Veroyatnost. i Mat. Statist., Kiev, 1972 (in Russian).

JOACHIM DOMSTA

INSTITUTE OF MATHEMATICS

UNIVERSITY OF GDAŃSK

WITA STWOSZA 57

80-952 GDAŃSK-OLIWA, POLAND

E-mail: MATJOD@UNIV.GDA.PL
FRANCISZEK GRABSKI

CHAIR OF MATHEMATICS

NAVAL ACADEMY

81-919 GDYNIA-OKSYWIE, POLAND 